\title{
Integrated Optical Elliptic Couplers: Modeling, Design, and Applications
}

\author{
Cailin Wei, Fokke Groen, Meint K. Smit, Member, IEEE, Ingrid Moerman, Member, IEEE, \\ Peter Van Daele, and Roel Baets, Member, IEEE
}

\begin{abstract}
This paper presents the modeling, design, experimental results and applications of integrated optical elliptic couplers. An approximate analytical model is presented and the design procedure is described. Some elliptic focusing and collimating structures have been experimentally tested and the results are in good correspondence with the design. The couplers have low losses, are polarization and lateral index contrast insensitive, compact, and easy to design and fabricate. The potential and limitations of the device are evaluated. Some applications of the elliptic couplers are also presented.
\end{abstract}

Index Terms - Add-drop filters, integrated optics, optical fiber coupling.

\section{INTRODUCTION}

$\mathbf{P}$ HOTONIC integrated circuits (PIC's) are expected to be of major importance for broadband telecommunication and optical information processing applications. For certain PIC functions, it is necessary to convert a beam guided by a channel waveguide into a collimated or focusing beam guided by a slab waveguide. In wavelength division multiplexing (WDM) systems, for example, a channel add/drop filter can be realized by using a collimated beam incident on a Bragg reflecting grating and using focusing elements to collect the reflected and the transmitted beams into channel waveguides. An expanded beam collimating element can also find applications in high-power devices to reduce the power density at a facet. For coupling light between semiconductor waveguides and single-mode fibers, beam focusing elements can be used to reshape the optical field profile in the film plane to get an optimal overlap with the field of the fiber [1].

Optical beam focusing and collimating functions in lateral direction (the layer plane) can in principle be realized by planar lenses [2], [3], adiabatic tapers and curved mirrors. However, each of these structures has its drawbacks. If the waveguide lateral index contrast is low, a planar lens has a large focal distance and therefore requires a lot of space. If, conversely, the lateral index contrast is high, the diffraction and reflection losses at the lens boundary may be high. Adiabatic tapers have been widely used, but tend to be long [4], [5]. Curved mirrors

Manuscript received August 13, 1996; revised January 17, 1997. This work was supported in part by the Belgian NFWO project "Optical switching in broad band communications: components and networks" and by the DWTCCOST240 project.

C. Wei, I. Moerman, P. Van Daele, and R. Baets are with the University of Gent-IMEC, INTEC Department, B-9000 Gent, Belgium.

F. Groen and M. K. Smit are with the Delft University of Technology, Delft, The Netherlands.

Publisher Item Identifier S 0733-8724(97)03541-X.

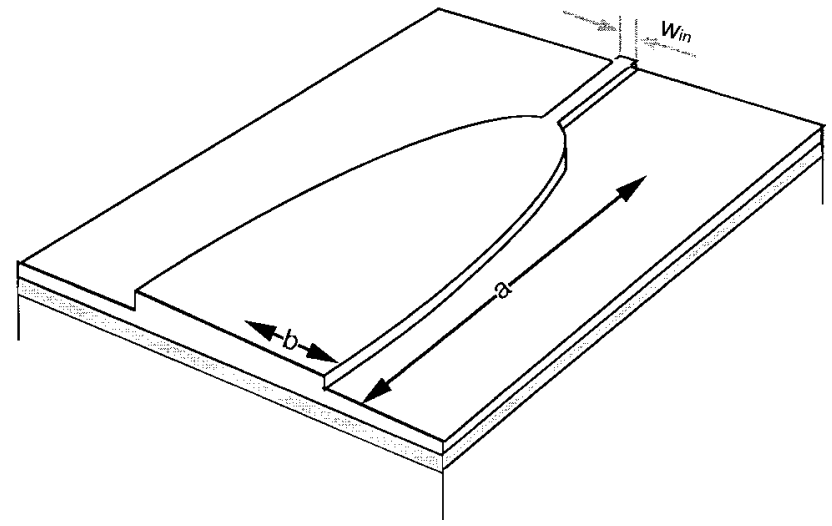

Fig. 1. Schematic figure of the elliptic coupler.

require a perfectly vertical etch profile and therefore depend on sophisticated technology.

As an alternative, elliptic beam couplers have been found to be able to focus or collimate beams in lateral direction [6]. They are compact, weakly sensitive to lateral index contrast and polarization [7] and have low losses [8]. These features allow a relaxed fabrication tolerance. In this paper, we present an analytical model to describe the properties of the elliptic couplers, the design rules, experimental results, and some applications.

\section{MODELING}

The elliptic coupler consists of an input waveguide followed by an elliptically shaped multimode section as shown schematically in Fig. 1. The coupler can be followed by an on-chip slab region (free diffraction in lateral direction) or by an offchip free space region in air (free diffraction in both transverse directions). By properly designing the lengths of the long and short axes $a$ and $b$ of the half ellipse, one can achieve a laterally focused beam with a desired beam width at a certain position or a laterally collimated beam.

The properties of elliptic couplers can be simulated by numerical methods such as the finite difference beam propagation method (FD-BPM). Such numerical methods however do not provide a clear understanding of the behavior of these devices and therefore optimization of the design is not obvious. To get a clear understanding and thereafter setting down a design rule, an analytical description is therefore necessary. However, there are no existing analytical formulas to describe the optical field produced by elliptic couplers accurately. We 


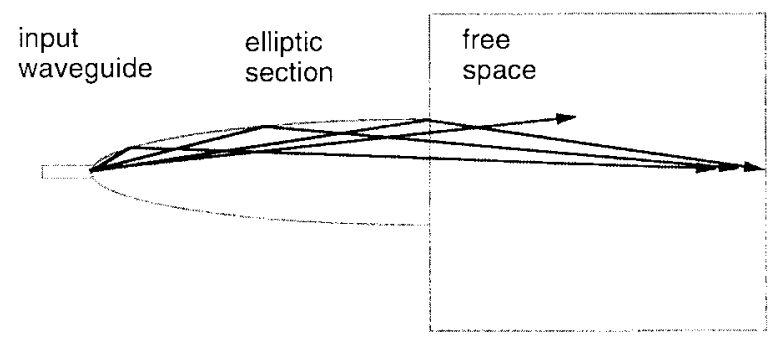

(a)

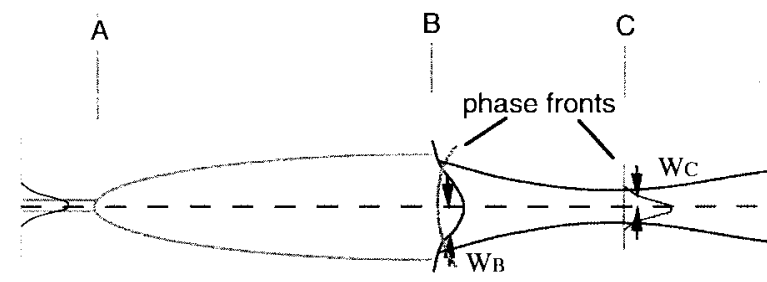

(b)

Fig. 2. Principle of operation of the elliptic coupler (a) by ray optics and (b) by Gaussian beam optics.

can understand the behavior of the elliptic couplers in two steps. First, from a ray optics point of view, if one puts a point light source at the left focal point of the half ellipse, the light rays toward the upper and lower boundaries will be reflected and focussed exactly on the right focal point while a small bundle of middle rays will propagate without reflection, as shown in Fig. 2(a). Second, from a wave optics point of view, the limited aperture of the converging beams will result in a focal point with a finite beam waist because of diffraction. Furthermore, the input field in reality is not a point source but rather the fundamental mode of an input waveguide, which will result in a shift of the position of the focal point. To model such a structure, different schemes have been tested. A first method is to treat the input beam as a superposition of three beams: an axial beam, an upper, and a lower beam. The upper and lower beams reflect off the upper and lower boundaries of the elliptic section and are transformed into converging beams while the axial middle beam propagates straight forward. As a first approximation one can use three Gaussian beams to approximately represent the above mentioned three beams. The interference of those three beams forms the total optical field in the region after the elliptic section. Reasonably good results have been obtained using this method, but there exists a drawback: there is no clear rule to determine the central directions of the upper and the lower Gaussian beams. It is found that the total field is rather sensitive to those directions and one has to use empirical formula to determine them.

A second method is to treat the two reflected converging beams and the middle diverging beam separately. We will describe this model in detail. Inspired by the ray optics picture, we assume the two reflected converging beams to form one converging beam with the right focal point of the ellipse as its phase front center. We then assume that this beam can be approximately represented by a converging Gaussian beam with its axis coincident with the waveguide axis. In this way we avoid the central direction issue of the reflected beams in the first method. At position $B$, the beam waist should be proportional to $b$ and the phase front radius should be proportional to $a$ as depicted in Fig. 2(b). We assume the Gaussian beam parameters to be expressed by the following formulas:

$$
\begin{aligned}
R_{B} & =-a \\
W_{B} & =\alpha b
\end{aligned}
$$

where $R_{B}$ is the radius of curvature of the phase front and $W_{B}$ is the half width of the beam (1/e point). $\alpha$ is a parameter close to 1 . As a first approximation to determine $\alpha$, we propose to calculate the $1 / e$ width of the fundamental mode of the waveguide of width $2 b$ and use this width for the Gaussian beam width. This leads to a value for $\alpha$, which depends on the lateral effective index contrast. If the region behind the coupler is air, the radius of curvature of the Gaussian beam will, at the interface, be reduced by a factor equaling the effective index of the waveguide

$$
R_{B, \text { air }}=-a / N_{\mathrm{eff}}
$$

With $R_{B}$ and $W_{B}$ as the starting point of the beam calculation, one can readily derive the field evolution in the following region by means of Gaussian beam $q$-parameter calculation. Given the elliptic coupler geometrical dimensions $a$ and $b$ which lead to $R_{B}$ and $W_{B}$ from (1) and (2) [or (2) and (3)], one arrives at

$$
\begin{aligned}
L_{C} & =\frac{-R_{B}}{1+R_{B}^{2}\left(\frac{\lambda}{\pi W_{B}^{2}}\right)^{2}} \\
W_{C} & =\frac{\lambda / \pi}{\sqrt{\left(\frac{W_{B}}{R_{B}}\right)^{2}+\left(\frac{1}{\pi W_{B}}\right)^{2}}}
\end{aligned}
$$

with $L_{C}$ the distance between the end of the coupler and the focal point and $W_{C}$ the waist of the focal point as shown in Fig. 2(b). $\lambda$ is the wavelength in this diffraction region. The beam parameter relationship at position $B$ and $C$ described by (4) and (5) are shown in Fig. 3. Given $W_{B}, L_{C}$ depends nonmonotonously on $R_{B}$ : one can find an $R_{B}$ value with maximal $L_{C}$. $W_{C}$ increases monotonously with $R_{B}$. On the other hand, given $R_{B}, L_{C}$ increases monotonously with $W_{B}$ while $W_{C}$ changes in a nonmonotonous way.

The justification of the model has been checked by comparing some of the calculated results from this model with results from FD-BPM calculations. The results are shown in Table I. The diffraction region is a wide slab region. The effective indexes used for the calculations are $N_{\text {core }}=3.31$ in the guiding region and $N_{\text {clad }}=1.0$ in the cladding region (deeply etched waveguide). In this case, the value is calculated to be 0.76 by using the method described earlier. Fig. 4 shows a calculated example for a device with $a=230 \mu \mathrm{m}$ and $b=8 \mu \mathrm{m}$ and with a slab free space region by using FDBPM. One can find that the focal point is a long region rather 


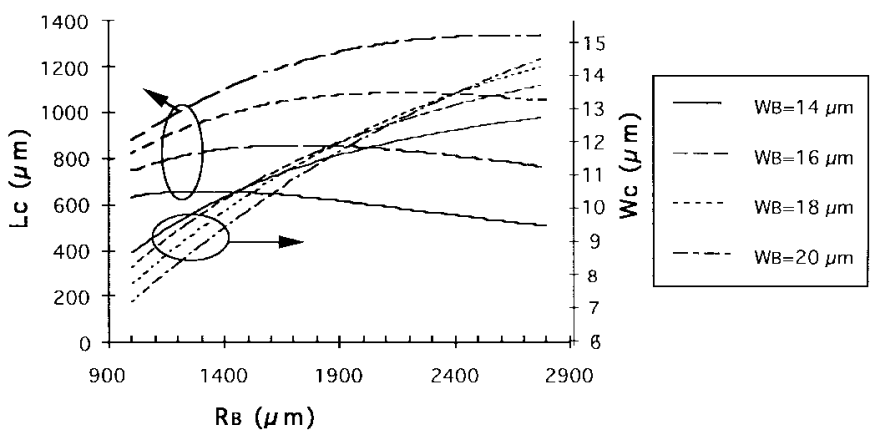

(a)

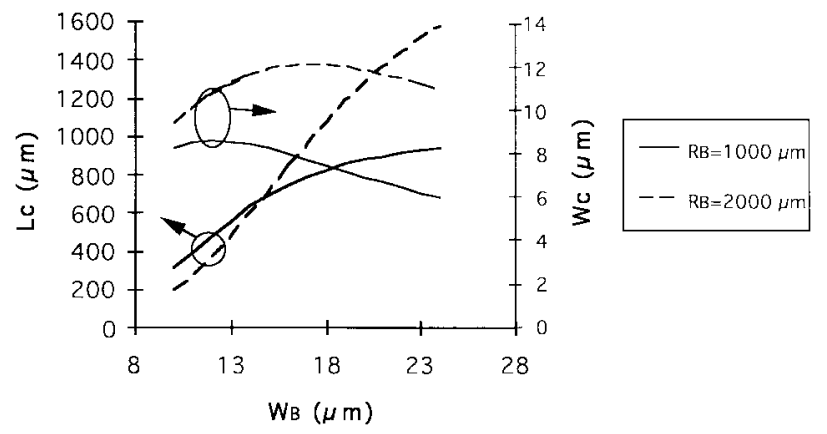

(b)

Fig. 3. Beam parameter relationship at position $\mathrm{B}$ and position $\mathrm{C}$ described by formulas (4) and (5).

TABLE I

Calculated Results by Using the Analytical Model and by Using THE FD-BPM. THE UsED WAVELENGTH Is $1.55 \mu \mathrm{mm}$ AND EFFECTIVE REFRACTIVE INDEXES FOR THE LATERAL GUIDING and Cladding Regions ARe 3.31 and 1.0, Respectively

\begin{tabular}{c|c|c|c|c|c|c}
\hline $\begin{array}{c}\mathrm{b} \\
(\mu \mathrm{m})\end{array}$ & $\begin{array}{c}\mathrm{W}_{\mathrm{B}} \\
(\mu \mathrm{m})\end{array}$ & $\begin{array}{c}\mathrm{R}_{\mathrm{B}}(=\mathrm{a}) \\
(\mu \mathrm{m})\end{array}$ & $\begin{array}{c}\mathrm{L}_{\mathrm{C}} \text { by this } \\
\text { model } \\
(\mu \mathrm{m})\end{array}$ & $\begin{array}{c}\mathrm{W}_{\mathrm{c}} \text { by this } \\
\text { model } \\
(\mu \mathrm{m})\end{array}$ & $\begin{array}{c}\mathrm{L}_{\mathrm{C}} \text { by FD- } \\
\mathrm{BPM} \\
(\mu \mathrm{m})\end{array}$ & $\begin{array}{c}\mathrm{W}_{\mathrm{C}} \text { by FD- } \\
\mathrm{BPM} \\
(\mu \mathrm{m})\end{array}$ \\
\hline 8 & 6.1 & 230 & 124 & 4.11 & $\sim 140$ & 4.0 \\
\hline 18 & 13.7 & 1000 & 608 & 8.50 & $\sim 600$ & 9.5 \\
\hline 20 & 15.2 & 2000 & 751 & 11.95 & $\sim 900$ & 12.5 \\
\hline 35 & 26.6 & 3000 & 2139 & 14.20 & $\sim 2100$ & 10.5 \\
\hline
\end{tabular}

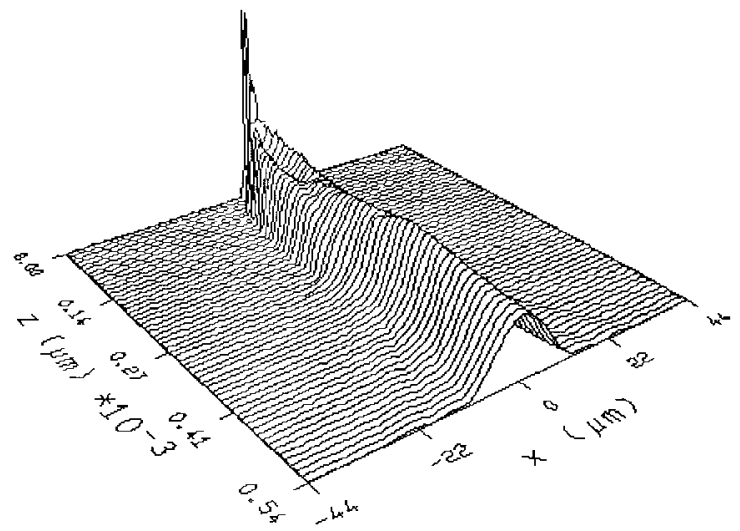

Fig. 4. Calculated optical amplitude field for a device of $a=230 \mu \mathrm{m}$ and $b=8 \mu \mathrm{m}$ with a slab diffraction region by using FD-BPM.

than a sharp point. In other examples similar phenomena can be seen. The differences between the analytical model and numerical method appearing in the examples in Table I are quite reasonable and therefore the model can form an useful design basis.
The differences between the analytical model and the numerical method are due to the Gaussian beam approximation and to the fact that the analytical model takes into account only the converging beam and not the diverging beam, while the numerical method includes everything. However the middle diverging beam has little influence on the position and the waist of the focal point. What the diverging beam does is to influence the profile of the total field through interference with the converging beam. The elliptic coupler can work properly only when the power fraction in the diverging beam is small. This power fraction, represented by $P_{\text {div }}$, can be calculated by using the following formula:

$$
P_{\text {div }}=\frac{2 \int_{0}^{\theta_{0}} \psi^{2}\left(f_{x}\right) d f_{x}}{\int_{-\infty}^{\infty} \psi^{2}\left(f_{x}\right) d f_{x}} .
$$

In the formula, $\psi\left(f_{x}\right)$ is the Fourier transform of the input field $\varphi(x), \theta_{0}=\operatorname{arctg}(b / a), f_{x}$ is the spatial frequency with $f_{x}=\sin \theta / \lambda$. When $P_{\text {div }}$ is small, its influence can be neglected.

\section{DESIGN}

Depending on the requirements for the output beam, one needs to determine the necessary coupler dimensions $a$ and $b$. For the design of the focusing structures, one can use (4) and (5) but in a reverse order, that is given $L_{C}$ and $W_{C}$ to calculate $R_{B}$ and $W_{B}$ using the rearranged formulas as below

$$
\begin{aligned}
\left(-R_{B}\right) & =L_{C}+\frac{1}{L_{C}}\left(\frac{\pi W_{C}^{2}}{\lambda}\right)^{2} \\
W_{B} & =\sqrt{W_{C}^{2}+\frac{L_{C}^{2} \lambda^{2}}{\pi^{2} W_{C}^{2}}} .
\end{aligned}
$$

One should keep in mind that (7) and (8) only represent the converging beam while the diverging middle beam is not included. In some of the focusing applications, this diverging beam represents losses. After an initial calculation of $R_{B}$ and $W_{B}$, one needs to estimate the power fraction in the diverging middle beam by using (6). If this power fraction is large, one needs to adjust the required $L_{C}$ and $W_{C}$ values and calculate $R_{B}$ and $W_{B}$ again until the middle beam is small. Another way to reduce the power in the middle beam is to minimize the waist of the input waveguide modal field by properly designing the width of the input waveguide (or by tapering it down). This weak middle beam requirement imposes a limitation to the obtainable $L_{C}$ and $W_{C}$ combinations.

As a special case of the focusing structure, a collimating structure can be designed (meaning: a structure in which a rather long beam waist of relatively constant width emerges directly from the coupler). This can be achieved by choosing an $(a, b)$ combination leading to a $W_{C}$ value close to $W_{B}$. In such a design a compromise must be chosen (which depends on coupler length) between length of collimated region and uniformity of its width. 


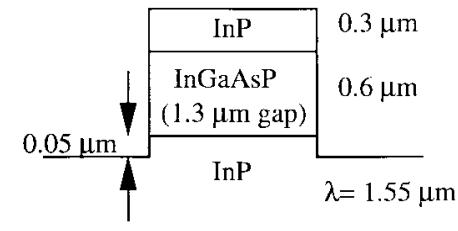

Fig. 5. Schematic view of the waveguide cross section.

\section{EXPERIMENT}

Based on the analytical model described in Sections II and III and numerical simulation results, some beam focusing and collimating structures have been designed and realized in InP. The waveguide cross sections used for the test chips for focusing and collimating structures are almost the same except for a minor thickness difference in the upper cladding layers. They consist of a $0.3 \mu \mathrm{m}$ (or $0.27 \mu \mathrm{m}$ ) InP top cladding layer and a $0.6 \mu \mathrm{m}$ thicuk InGaAsP core layer with $1.3 \mu \mathrm{m}$ gap, grown on an InP substrate and is deeply etched through the guiding layer, as shown in Fig. 5.

\section{A. Focusing Structures}

Two types of structures were tested. In the first type the half ellipse ends on a semiconductor slab region with the same transverse cross section as the ellipse. A number of a and $\mathrm{b}$ value combinations have been tested. For one of them, a device with $a=230 \mu \mathrm{m}$ and $b=8 \mu \mathrm{m}$ and a slab region of $150 \mu \mathrm{m}$ long (where the chip ends), results are shown. Fig. 6 shows its focal point at the chip end measured by a camera where the full width at half maximum (FWHM) of the intensity field is $6 \mu \mathrm{m}$. The width of the input waveguide is $2 \mu \mathrm{m}$. The waist of the focussed point is in good agreement with the calculated value. Other tested structures also have good correspondence with the designed values. In the second type of structure the half ellipse ends directly on an air facet. Again, for the device with $a=230 \mu \mathrm{m}$ and $b=8 \mu \mathrm{m}$ as example, the field (intensity) evolution in air behind the coupler is measured by a camera scanned in lateral direction and the result is shown in Fig. 7 where one can find the the focal point position and its beam waist. Those measured results have again very good correspondence with the calculated values.

\section{B. Collimating Structures}

To test the beam collimating properties of the elliptic couplers, two types of structures were designed and fabricated. A first type of structure is for checking the optical field evolution in the slab region. This is done by using an identical elliptic section but different slab region lengths. Several different elliptic collimators were tested with different $a$ and $b$ value combinations. Light was launched into the input waveguide and a camera was used to measure the optical intensity distributions on the facet. The measurement results suggest that the beam waist change is small and the results are in very good correspondence with the designed values [8].

A second type of structure is for checking the collimator losses. To do this, some collimator pairs were designed and fabricated. For comparison, straight waveguides and parabolic collimators have also been fabricated. The structures were

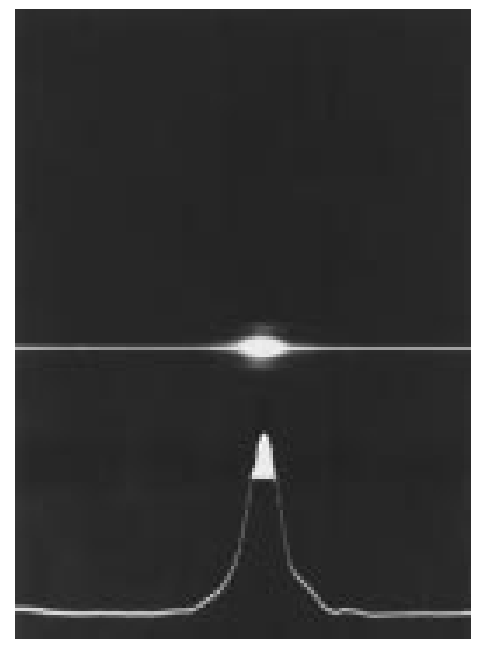

Fig. 6. Photograph of the optical intensity distribution at the focal point for the device with $a=230 \mu \mathrm{m}$ and $b=8 \mathrm{~m}$.

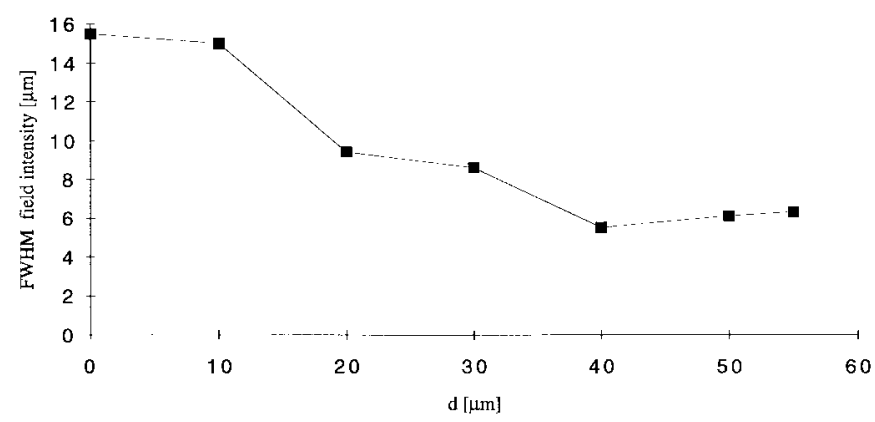

Fig. 7. Beam waist (FWHM) of intensity as a function of the propagated distance in air for the structure of Fig. 1b. The measurements were performed for a device with $a=230 \mu \mathrm{m}$ and $b=8.0 \mu \mathrm{m}$.

designed such that after the ellipse, the phase curvature of the optical field in the slab region evolve from slightly converging to flat and then to slightly diverging in the designed propagation region. A second elliptic coupler follows and allows the optical field to be imaged onto the receiving waveguide. The realized collimator pairs are shown in Fig. 8. In comparison a parabolic collimator can also form a collimated beam in the slab region, but the beam phase front will be from flat to diverging relatively quick and this will result in a broader beam.

To characterize the device losses, the Fabry-Perot method was used [9]. Since the F-P method depends on knowledge of the facet reflectivity, we calculated the power reflection coefficient for the TE-mode. With the resulting value of $38 \%$, the overall loss for the elliptic coupler pairs, for a structure with $a=1000 \mu \mathrm{m}$ and $b=18 \mu \mathrm{m}$ and a $1000 \mu \mathrm{m}$ long slab region, was found to be $1.18 \mathrm{~dB}$, to be compared with $1.09 \mathrm{~dB}$ loss for the straight waveguide and $3.05 \mathrm{~dB}$ loss for the parabolic collimator pairs. The straight waveguide used here has the same width as the collimator input and output waveguide and the devices have the same length.

\section{APPLICATION}

As beam shaping elements, elliptic couplers can find applications when beam expansion, focusing or collimation is 


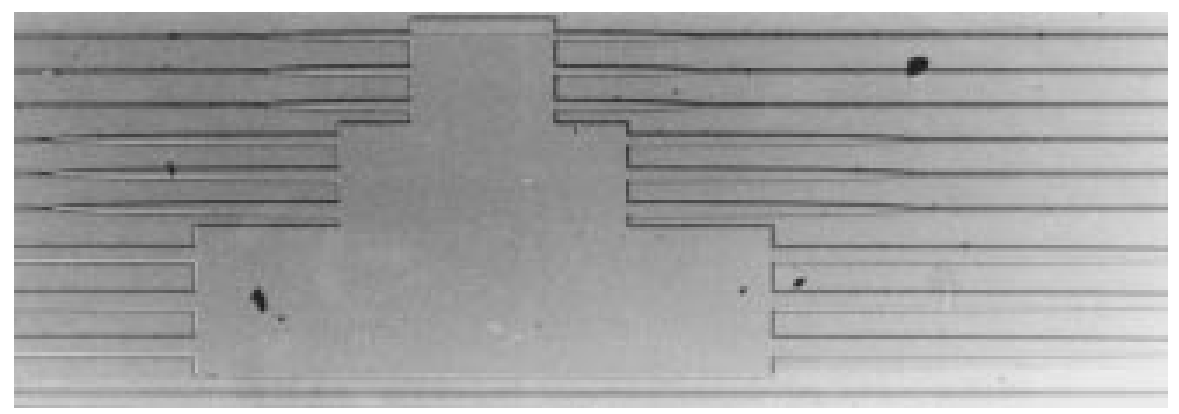

Fig. 8. Photograph of the realized collimator pairs.

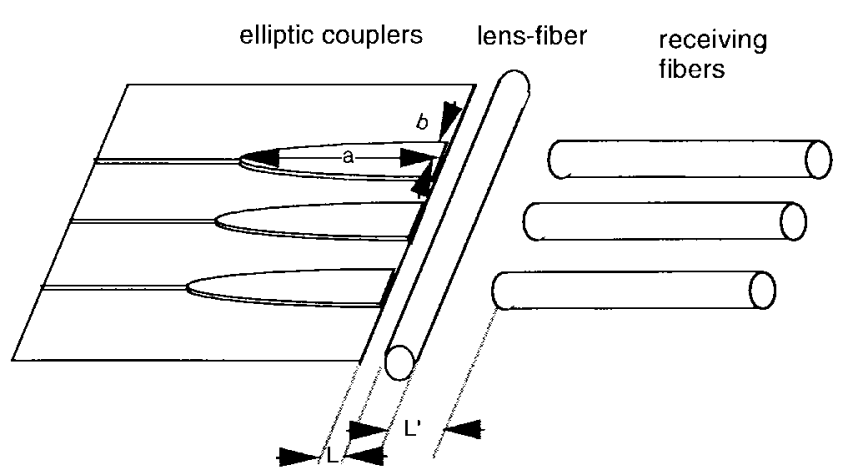

Fig. 9. Schematic coupling structure between waveguide array and SMF array.

required. As the device is based on total internal reflection, it is relatively insensitive to lateral refractive index contrast and therefore has good compatibility to many types of materials and technologies. We will describe its application in a waveguide array to single mode fiber array coupling scheme and its application in waveguide add/drop filters.

\section{A. Fiber Array Coupling}

Many chip to fiber coupling structures have been proposed and tested. Most of them use either a tapered section in the chip side or a lensed structure in the fiber side [10]. Elliptic couplers offer a new alternative well adapted to fiber array coupling [1]. It does not require additional process steps compared to butt coupling. A schematic layout of the coupling structure using elliptic couplers is shown in Fig. 9. In each channel a waveguide enters an integrated elliptic coupler which adapts the input field and focusses it laterally into a single mode fiber at a certain distance away from the chip. In vertical direction, the diverging beam from the chip is converged and the field profile is magnified into single mode fiber by a cylindrical lens which is a piece of standard fiber.

The designing procedure is not complicated. The standard fiber has a given set of parameters: refractive index $n=1.5$ and cladding diameter $D=125 \mu \mathrm{m}$. Given the FWHM of the waveguide field in vertical direction, one can calculate the required lens magnification $M$ for an optimum field overlap with a receiving fiber, from which the distances $L$ and $L^{\prime}$ shown in Fig. 9 are obtained. Then one can design the $a$ and $b$ values of the elliptic coupler by using the formulas presented in Section III to optimize the coupling in lateral direction.
A test chip was fabricated using a waveguide cross section similar to the one described in section 4 based on InP material. The designed dimensions of the ellipse are $a=3000 \mu \mathrm{m}$ and $b=35 \mu \mathrm{m}$ and the distances are $L=50 \mu \mathrm{m}$ and $L^{\prime}=500 \mu \mathrm{m}$. The calculated coupling efficiency in lateral direction is $83 \%$ and $70 \%$ in vertical direction using the Gaussian beam overlap method. The total coupling efficiency is $\eta=\eta_{/ /} \times \eta_{\perp}=58 \%$. This value did not take into account the reflection losses by the fiber surfaces. There is another source of losses which need to be taken into account: the air radiation loss. For the waveguide structure used here, a part of the ray bundle (with large angles to the optical axis) can not be collected by the lens-fiber and radiated into air directly. To calculate the losses of the air radiation, the radiated far field from the waveguide has been calculated. The results are as follows. The air radiation loss is $13.4 \%$. Of the rays reaching the first surface of the lens-fiber, 7.67 and 3\% power is reflected at this surface for TE and TM polarizations, respectively. Take into account the two interfaces of the lens-fiber and the two interfaces of the receiving fiber, the calculated total reflection losses for TE and TM waves are 21.5 and $13.3 \%$, respectively. Without putting antireflection (AR) coatings on the surfaces of the lens-fiber and the facets of the receiving fiber, $25 \%$ coupling efficiency is achieved experimentally. We believe with AR coatings and a further optimized structure, an average (TE and TM) $40 \%$ coupling efficiency is achievable. Since this scheme is based on flat fiber ends, one can expect the uniformity between channels and reproducibility to be good.

This scheme can also be used to couple light from waveguides of semiconductor amplifier arrays with slanted facet into single mode fibers. The wide beam at the facet allows the slanting angle (to achieve low reflectivity) to be very small.

\section{B. Add/Drop Filters}

In WDM systems the channel add/drop filters are essential since they offer the possibility to get access to each of the wavelength channels while leaving other channels untouched. Using elliptic couplers as collimating and focusing elements, together with an on-chip Bragg grating reflector which can be fabricated, e.g., by holographic technology, one can construct integrated channel add/drop filters in an efficient way. One of the proposed configurations is shown in Fig. 10. Coupler 1 generates a collimated input beam and coupler 2 receives the reflected beam from the grating while coupler 3 receives the transmitted beam. Coupler 4 is for adding the channel with 


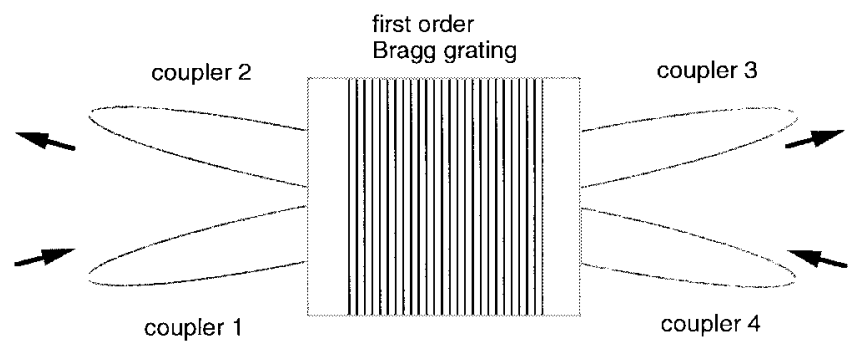

Fig. 10. Schematic configuration of a planar channel add/drop filter.

the droped wavelength to arm 3. The spectral properties of the device are mainly determined by the grating while the loss properties are determined mainly by two factors: the losses of the couplers and the losses due to imperfections of the grating. According to the coupler loss characteristics described in Section IV, one can expect a very low device losses.

\section{CONCLUSION}

In this paper, we presented an analytical model to approximately describe the beam shaping function of the elliptic couplers. The design procedure is also presented. Those formulas can form a basis for quick device design. Based on those calculated data, one can use numerical tools to refine the design.

The device properties as focusing and collimating structures have been tested experimentally. The measurement results are in good correspondence with the designed values. The elliptic couplers have been tested in fiber array coupling applications. Because of its advantageous features in terms of losses, polarization dependence, index contrast sensitivity, fabrication tolerance, device size, bandwidth and so on, it is a good alternative in many applications to etched lenses on chip. One limitation to the device is that the power fraction in the middle beam should be kept low.

\section{ACKNOWLEDGMENT}

The authors would like to acknowledge A. Jarmolik, L. Vanwassenhove of the University of Gent, and C. G. M. Vreeburg of the Technical University of Delft for their contributions to the device fabrication.

\section{REFERENCES}

[1] C. Wei , A. Jarmolik, F. H.Groen, C. G. M. Vreeburg, L. Vanwassenhove, I. Moerman, P. Van Daele, and R. Baets, "New waveguide-fiber coupling method for fiber arrays based on integrated elliptic couplers," in 22nd European Conf. Optical Commun. (ECOC'96), OSLO, 1996.

[2] J.-M. Verdiell, M. A. Newkirk, T. L. Koch, R. P. Gnall, U. Koren, B. I. Miller, and L. L. Buhl, "Aspheric waveguide lenses for photonic integrated circuits," Appl. Phys. Lett., vol. 62, no. 8, pp. 808-810, Feb. 22, 1993.

[3] T. J. Su and C. C. Lee, "An embedded waveguide lens with antireflection layer", IEEE Photon. Technol. Lett., vol. 6, pp. 89-91, Jan. 1994.

[4] I. S. Duport, P. Benech, and R. Rimet, "New integrated-optics interferometer in planar technology," Appl. Opt., vol. 33, no. 25, pp. 5954-58, Sept. 1994.

[5] J. D. Love, W. M. Henry, W. J. Stewart, R. J. Black, S. Lacroix, and F. Gonthier, "Tapered single-mode fibers and devices, Part 1: Adiabaticity criteria," Inst. Elec. Eng. Proc. J., vol. 138, no. 5, pp. 343-354, 1991.
[6] C. Wei, J. Haes, I. Moerman, R. Baets, and M.K. Smit, "Elliptic waveguide beam focusing and collimating elements in InP: analysis and experiment," Electron. Lett., vol. 31, no. 25, pp. 2168-2169, 1995.

[7] C. Wei, J. Haes, G. Dobbelaere, P. Demeester, and R. Baets, "Elliptic beam converters on InP: design and experiment," in Proc. 7th European Conf. Integr. Opt., Apr. 3-6, 1995, Delft, The Netherlands, pp. 193-196.

[8] C. Wei , F. H.Groen, C. G. M. Vreeburg, P. Demeester, and R. Baets, "Very low loss compact waveguide beam collimators on InP," Electron. Lett., to be published.

[9] R. G. Walker, "Simple and accurate loss measurement technique for semiconductor optical waveguide," Electron. Lett., vol. 21, no. 13, pp. 581-583, June 1985

[10] I. Moerman, M. D'Hondt, W. Vanderbauwhede, J. Blondelle, G. Coudenys, P. van Daele, and P. Demeester, "III-V semiconductor waveguiding devices using adiabatic tapers," Microelectron. J., vol. 25, pp. 675-690, 1994.

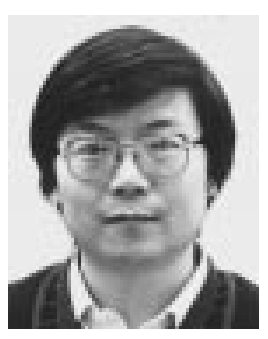

Cailin Wei received the Diploma degree in physics from Baotou Teacher's College, China, in 1980. $\mathrm{He}$ received the M.Sc. degree in optics from Xian Institute of Optics and Fine Mechanics, Chinese Academy of Sciences, China, in 1988. He is currently a Ph.D. candidate in applied physics in the University of Gent, Gent, Belgium.

From 1980 to 1985, he worked in the Department of Physics of Baotou Teacher's College as a Lecturer Assistant. In 1985, he joined the Xian Institute of Optics and Fine Mechanics, Chinese Academy of Sciences, as a Researcher. Since 1992, he has been with the Department of Information Technology (INTEC) of the University of Gent as a Research Assistant. He has worked in the field of integrated optical elements and optical fiber sensors. His main interests are the modeling, design and testing of optoelectronic devices, and optical fiber sensors.

Fokke Groen received the M.Sc. degree in electrical engineering from Eindhoven University of Technology, Eindhoven, The Netherlands, in 1965.

Until 1972, he was concerned with optical filtering and system theory at the Department of Electrical Engineering of this University. In 1972, he joined the Optics Research Group of the Department of Applied Physics, Delft Technical University, Delft, The Netherlands. Since then, his interests have been in lasers, nonlinear optics, digital holographic and speckle interferometry. During the last decade, he was concerned with integrated optics on the basis of InP, mainly for telecom applications, with emphasis on process technology for passive waveguides, switches, and wavelength selective devices.

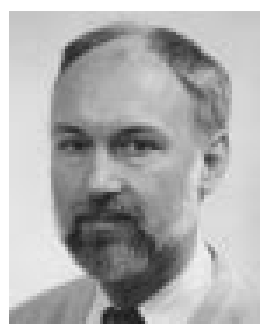

Meint K. Smit (A'93) was born in Vlissingen, the Netherlands, in 1951. He received the degree in electrical engineering at the Delft University of Technology, Delft, The Netherlands. During 1969-1974, he received the Master's and Ph.D. degrees (both with honors).

In 1974, he started as a Research Scientis with the NIWARS (Netherlands Interdepartmental Working Group on Application of Remote Sensing Technology. He joined the Delft University of Technology in 1976 as an Assistent Professor with responsibility for research in Microwave Remote Sensing and FMCW radar development. He switched to optical communication in 1981, where he had set-up facilities for development of silicon-based integrated optical devices. He invented the phased-array wavelength demultiplexer and worked on multimode interference (MMI) couplers, optical switches, measurement and characterization of electro-optical devices, and development of computer-aided design tools. Since 1986, his focus has shifted to InP-based semiconductor devices. From 1991 to 1992, he was on leave at the Institute of Quantum Electronics, ETH Zurich, Zurich, Switzerland, where he worked on development of a fast and compact polarization independent optical switch.

Dr. Smit is a member of IEEE/LEOS and the Netherlands Electronics and Radio Society (NERG). 


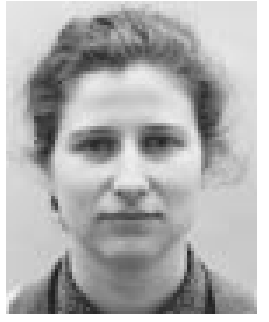

Ingrid Moerman (M'96) was born in Gent, Belgium, in 1965. She received the degree in electrical engineering and the Ph.D. degree from the University of Gent, Gent, Belgium, in 1987 and 1992, respectively.

Since 1987, she has been with the Interuniversity Micro-Electronics Centre (IMEC)at the Department of Information Technology of the University of Gent, where she is active in the epitaxial growth of InGaAsP/InP and $\mathrm{In}(\mathrm{Al}) \mathrm{GaAs} / \mathrm{GaAs}$ laser diodes and waveguiding devices. In 1997, she will be a permanent member of the Research Staff at IMEC, where she will coordinate the research on the epitaxial growth of III-V opto-electronic devices.

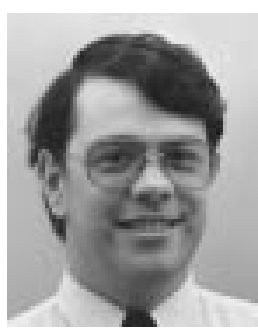

Peter Van Daele was born in 1961 and received the $\mathrm{Ph} . \mathrm{D}$. degree in electrical engineering in 1988 from the University of Gent, Gent, Belgium.

He became a permanent Member of Staff from the Interuniversity Micro-Electronics Centre (IMEC) at the Department of Information Technology of the University of Gent, where he is responsible for the research on processing and packaging of IIIV optoelectronic devices. In 1993 he also became part-time Professor. He is author or coauthor of about 200 publications in the field of optoelectronic

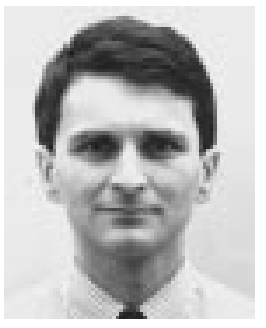

Roel Baets (M'88) received the degree in electrical engineering from the University of Gent, Gent, Belgium, in 1980. He received the M.Sc. degree in electrical engineering from Stanford University in 1981 and the Ph.D. degree from the University of Gent in 1984.

Since 1981, he has been with the Department of Information Technology (INTEC) of the University of Gent. Since 1989, he has been a Professor in the Engineering Faculty of the University of Gent. From 1990 to 1994, he has also been a part-time Professor at the Technical University of Delft, Delft, The Netherlands. He has worked in the field of III-V devices for optoelectronic systems. With over 100 publications and conference papers he has made contributions to the modeling of semiconductor laser diodes, passive guided wave devices and to the design and fabrication of OEIC's. His main interests now are the modeling, design and testing of optoelectronic devices, circuits and systems for optical communication and optical interconnect.

Dr. Baets is a member of the Optical Society of America, IEEE-LEOS, SPIE, and the Flemish Engineers Association. He has been member of the program committees of OFC, ECOC, IEEE Semiconductor Laser Conference, ESSDERC, CLEO-Europe and the European Conference on Integrated Optics.

components and technology. 